\title{
Association Between Abnormal Glycemic Phenotypes and Microvascular Complications of Type 2 Diabetes Mellitus Outpatients in China
}

This article was published in the following Dove Press journal: Diabetes, Metabolic Syndrome and Obesity: Targets and Therapy

\author{
Guangxu Liu $\mathbb{D}^{1,2}$ \\ Jingtao Dou ${ }^{3,4}$ \\ Deqiang Zheng ${ }^{1,2}$ \\ Jie Zhang ${ }^{1,2}$ \\ Meiping Wang ${ }^{1,2}$ \\ Wei $\mathrm{Li}^{1,2}$ \\ Jing Wen ${ }^{1,2}$ \\ Juming $\mathrm{Lu}^{3}$ \\ Linong $\mathrm{Ji}^{5}$ \\ Yan $\mathrm{He}^{1,2}$
}

'Department of Epidemiology and Biostatistics, School of Public Health, Capital Medical University, Beijing, People's Republic of China; ${ }^{2}$ Beijing Municipal Key Laboratory of Clinical Epidemiology, Beijing, People's Republic of China; ${ }^{3}$ Department of Endocrinology, Chinese PLA General Hospital, Beijing, People's Republic of China; ${ }^{4}$ Department of Endocrinology, Hainan Branch of Chinese PLA General Hospital, Sanya, People's Republic of China; ${ }^{5}$ Department of Endocrinology and Metabolism, Peking University People's Hospital, Beijing, People's Republic of China
Correspondence: Yan He; Jingtao Dou Email yanheI220@I26.com;

jingtaodou@163.com
Purpose: The objective of this study was to investigate the association of abnormal glycemic phenotypes with microvascular complications in type 2 diabetes patients.

Participants and Methods: A total of 24,266 participants who were from the multicenter cross-sectional survey of China National HbA1c Surveillance System across China were included in the present study. Diabetes patients with abnormal glucose were divided into three groups according to phenotype: isolated fasting hyperglycemia (IFH), isolated postprandial hyperglycemia (IPH), or combined hyperglycemia $(\mathrm{CH})$. The main outcomes were isolated diabetic retinopathy, isolated diabetic nephropathy, and combined diabetic retinopathy with nephropathy. Multivariate logistic regression was used to assess the association of abnormal glycemic phenotypes with microvascular complications.

Results: The $\mathrm{CH}$ phenotype had the highest prevalence of isolated diabetic retinopathy, isolated diabetic nephropathy and combined diabetic retinopathy with nephropathy, followed by IPH. Multivariate analysis showed that the $\mathrm{CH}$ phenotype was associated with the highest risk of isolated diabetic retinopathy (OR: $1.20,95 \% \mathrm{CI}: 1.02-1.41$ ), isolated diabetic nephropathy (OR: 1.59, 95\% CI: 1.27-2.01) and combined diabetic retinopathy with nephropathy (OR: 1.93, 95\% CI: 1.44-2.59). More importantly, participants with IPH phenotype also showed significantly higher risks of isolated diabetic retinopathy (OR: 1.16, 95\% CI: 1.05-1.28), isolated diabetic nephropathy (OR: 1.37, 95\% CI: 1.09-1.37) and combined diabetic retinopathy with nephropathy (OR:1.64, 95\% CI: 1.21-2.21) compared to the IFH phenotype. After stratifying by age, sex, diabetes duration and BMI, the higher risks of isolated diabetic retinopathy, isolated diabetic nephropathy and combined diabetic retinopathy with nephropathy were confirmed in IPH phenotype group, compared to the IFH phenotype group.

Conclusion: Diabetic patients with IPH phenotype had higher risks of isolated diabetic retinopathy, isolated diabetic nephropathy and combined diabetic retinopathy with nephropathy compared with the participants phenotype of IFH, but lower than the phenotype of $\mathrm{CH}$.

Keywords: abnormal glycemic phenotype, diabetic nephropathy, diabetic retinopathy, type 2 diabetes mellitus

\section{Introduction}

Type 2 diabetes mellitus (T2DM) has become a substantial threat to human health in the past decades. According to the estimation from World Health Organization (WHO), the worldwide number of diabetic people would exceed 366 million by 2030. ${ }^{1}$ In China, the prevalence of diabetes has also risen from $0.67 \%$ in 1980 to $10.9 \%$ in $2013^{2}$ and the number is expected to exceed 129.7 million by the year 
2030. ${ }^{3}$ T2DM could lead to microvascular complications which include diabetic nephropathy and diabetic retinopathy. More than $40 \%$ of T2DM patients have diabetic nephropathy ${ }^{4}$ and $18 \% \sim 43 \%$ patients have diabetic retinopathy. ${ }^{5}$ Furthermore, nephropathy or retinopathy is a major risk factor for end-stage renal disease $\mathrm{e}^{4}$ and vision $\operatorname{loss}^{6}$ for the diabetic population, which makes diabetes mellitus a most alarming public health problem.

T2DM is characterized by chronic abnormal glucose metabolism which includes abnormal fasting plasma glucose (FPG) and/or abnormal postprandial plasma glucose (PPG). Many previous studies focused on the association between glucose metabolism and risk of microvascular disease. Some findings showed that elevated FPG or PPG were related to the onset risk of retinopathy and nephropathy. ${ }^{7-9}$ However, it is inconsistent regarding which type of abnormal glucose metabolism is associated with higher risk of nephropathy and retinopathy. A clinical trial of 151 Japanese T2DM patients showed that only PPG was significantly correlated with the progression of retinopathy and is a stronger predictive factor than $\mathrm{FPG},{ }^{10}$ whereas another study showed that there was no difference in the predictive value of retinopathy between FPG and PPG. ${ }^{11}$ For nephropathy, a cross-sectional study showed that FPG was a factor related to positive proteinuria and impaired glomerular filtration rate, ${ }^{12}$ but one study based on the Systolic Blood Pressure Intervention Trial (SPRINT) found that FPG was not associated with the development of kidney disease. ${ }^{13}$ The data from a cohort study demonstrated that PPG was a more important determinant of onset and development of nephropathy in T2DM patients. ${ }^{14}$ Nevertheless, McCance et al reported PPG was equally predictive of nephropathy in T2DM. ${ }^{11}$ The existing evidence indicates a certain association between the elevation of fasting or postprandial glucose and increasing risk of microvascular disease, however, the type of hyperglycemia that has a larger effect on microvascular complications has not been uniformly addressed. The inconsistency may be explained by the difference in sample size and ethnic group in different studies. To our knowledge, few studies were performed to investigate the contribution of FPG and PPG to the risk of retinopathy and nephropathy in a Chinese population.

To clarify these issues, we extracted a T2DM population to assess the association between abnormal glycemic phenotypes and the risk of microvascular diseases in a Chinese population. This population was collected from a nationwide multicenter study of China National $\mathrm{HbAlc}$ Surveillance System (CNHSS).

\section{Participants and Methods Study Population}

The CNHSS project was a cross-sectional large population survey conducted from April to June in 2013 in Mainland China, which was launched by the Chinses Diabetes Society. The CNHSS was established to monitor glycemic control among adult patients with T2DM. The survey recruited a total of 238,639 participants with T2DM from 602 hospitals located in all provincial administrative regions (except Guangxi Zhuang Autonomous Region and Tibet) in China mainland. The inclusion and exclusion criteria have been described previously. ${ }^{15}$ Briefly, the inclusion criteria were: 1) T2DM outpatients who were 18 years or more; 2) diagnosed by 1999 WHO's criteria; ${ }^{16}$ 3) treated with antidiabetic agents, diet or lifestyle therapy. The exclusion criteria included: 1) diabetes secondary to other diseases; 2) retinopathy or nephropathy diagnosed prior to diabetes diagnosis; 3) being treated with Chinese herbal medicine only; 4) being pregnant or breast-feeding; and 5) unconsciousness or being unable to communicate. In each workday during the survey period, the first consecutive 7 participants who entered each hospital's endocrinology outpatient department and had medical records and met the eligibility criteria, were invited to participate in involved hospitals, until 400 participants were recruited in each involved hospital during the whole recruitment period unless the recruitment period had ended. In order to select a representative population whose key features were similar to those of the national T2DM population, ${ }^{2}$ we randomly selected the sample using a threestage stratification-random clustering sampling method, based on the distribution proportion of T2DM participants in geographic distribution, economic status and age. Furthermore, the T2DM participants whose date of diagnosis was later than the date of occurrence of microvascular disease were excluded. Finally, 24,266 participants were included in this analysis. All the T2DM participants provided written informed consent before entering the study. Ethics approval was obtained from the Ethics Committee for Clinical Research of the People's Liberation Army General Hospital, and also accepted by all the participating hospitals.

\section{Data Collection}

Information such as age, sex, height, weight, and blood pressure, was collected using a structured form through 
face-to-face interview by trained fieldworkers or measured through physical examination in the involved hospitals. Clinical characteristics were obtained by checking the medical records, which included glucose level at diagnosis of diabetes, the history of disease, as well as diagnosis date. Participants were required to confirm again whether they were diagnosed with any concomitant diseases or diabetes complications, including hypertension, coronary heart disease, cerebrovascular disease, dyslipidemia, diabetic retinopathy, and diabetic nephropathy, and diagnosis date, and this was checked via medical records of local hospital or diagnosis certificate by secondary or tertiary hospital. They were also required to report treatment information of management of T2DM, including the use of oral antidiabetic drugs (OADs), and different types of insulin, as well as diet or lifestyle therapy. Heights and weights were measured, in light clothing, with the use of standardized stadiometers and scales, respectively. Blood pressure was measured on the right arm using a standard mercury sphygmomanometer or an electronic sphygmomanometer with the subjects resting for at least 5 minutes in a sitting position. All laboratory examinations for FPG, 2-hour PPG (2hPPG), $\mathrm{HbA1c}$ and lipids such as triglyceride, total cholesterol and low-density lipoprotein, were performed in local hospital during the survey period. Blood sample was collected in local hospital after overnight $10 \mathrm{~h}$ fasting for biochemical analyses. The 2hPPG was measured after the participants had completed the $75 \mathrm{~g}$ oral glucose tolerance test. All designated researchers were trained on basics of T2DM, procedures of data collection and entry, and details of the face-to-face interview. In addition, quality supervisors checked the reliability of the data by randomly re-interviewing some participants. All the data were entered and uploaded to the central database of CNHSS by experienced fieldworkers.

\section{Definitions of Key Variables}

According to FPG and $2 \mathrm{hPPG}$ level at diagnosis of diabetes, the abnormal glycemic phenotype included isolated fasting hyperglycemia (IFH) defined as $\mathrm{FPG} \geq 7.0 \mathrm{mmol} / \mathrm{L}$ and $2 \mathrm{hPPG}<11.1 \mathrm{mmol} / \mathrm{L}$, isolated postprandial hyperglycemia (IPH) defined as $2 \mathrm{hPPG} \geq 11.1 \mathrm{mmol} / \mathrm{L}$ and $\mathrm{FPG}<7.0$ $\mathrm{mmol} / \mathrm{L}$ and combined hyperglycemia $(\mathrm{CH})$ defined as $\mathrm{FPG} \geq 7.0 \mathrm{mmol} / \mathrm{L}$ and $2 \mathrm{hPPG} \geq 11.1 \mathrm{mmol} / \mathrm{L}$. The economic development level was defined as undeveloped region, intermediately developed and developed region based on the Gross Domestic Product (GDP) of each province in 2013. The blood pressure control target was defined as systolic blood pressure $<140 \mathrm{mmHg}$ and diastolic blood pressure $<90 \mathrm{mmHg}$. HbAlc control target was defined as $<7.0 \%$ (53 mmol $/ \mathrm{mol})$. The diagnosis of microvascular diseases was confirmed by the medical recordings. Diabetic retinopathy was diagnosed by ophthalmologist based on typical changes of retinopathy on funduscopic examination due to diabetes, including background, pre-proliferative, proliferative or maculopathy. Diabetic nephropathy was defined as having persistent proteinuria, such as urinary albumin excretion rate $\geq 20 \mu \mathrm{g} /$ min or urinary albumin $\geq 30 \mathrm{mg} / 24 \mathrm{~h}$ after excluding other causes of kidney damage, urinary system infection, and blood in urine. In this study, the outcomes mainly included isolated diabetic retinopathy (IDR) defined as only diabetic retinopathy not diabetic nephropathy, isolated diabetic nephropathy (IDN) defined as only diabetic nephropathy not diabetic retinopathy and combined diabetic retinopathy and nephropathy $(\mathrm{CRN})$ defined as both of them.

\section{Statistical Analysis}

Continuous variables of clinical characteristics were presented as mean with standard deviation or median with interquartile range (IQR). Categorical variables were described as frequency with proportion. Analysis of variance (ANOVA) test or Kruskal-Wallis rank test was applied to compare continuous variables among T2DM participants with different phenotypes. The Bonferroni or Nemenyi test was used for pairwise comparison among three groups. Chi-squared test and Fisher's exact test were used to compare categorical variables.

Unconditional logistic regression analysis was used to estimate odds ratios (ORs) and $95 \%$ confidence intervals (CI) for the association between abnormal glycemic phenotypes (IFH group as reference) and microvascular complications, which were adjusted for potential confounding factors by four step forward multivariable-adjusted models. Model 1 was no adjustment of covariates. Model 2 was adjusted for age and sex. Model 3 was adjusted for age, sex, and diabetes duration. Model 4 was adjusted for age, sex, diabetes duration, BMI, self-monitoring of blood glucose, diabetes treatment, the target of blood pressure control, the target of lipid control, HbAlc and economic development level. Kaplan-Meier analysis was used to plot cumulative incidence of microvascular disease of different abnormal glycemic phenotypes. Then the Log rank test was used to compare the difference of cumulative 
incidence among abnormal glycemic phenotypes. When we compared the cumulative incidence of microvascular diseases among abnormal glycemic phenotypes, the crosssectional survey was transformed into a retrospective cohort. The follow-up time was calculated as time in years from the date of diagnosis of diabetes to the date of occurrence of the disease or the date of the survey, whichever came first.

All statistical analyses were performed by the statistical analysis system (SAS) version 9.4 (SAS Institute Inc., Cary, NC, USA). A two-sided value of $\mathrm{P}<0.05$ was considered statistically significant. Kaplan-Meier plot and forest plot

Table I Characteristics of T2DM Participants with Different Abnormal Glycemic Phenotypes

\begin{tabular}{|c|c|c|c|c|c|c|c|c|}
\hline Characteristics & $\begin{array}{l}\text { Overall } \\
(n=24,266)\end{array}$ & $\begin{array}{l}\text { IFH } \\
(n=4493)\end{array}$ & $\begin{array}{l}\mathrm{IPH} \\
(\mathrm{n}=10,453)\end{array}$ & $\begin{array}{l}\mathrm{CH} \\
(n=9320)\end{array}$ & $\mathbf{P}$ & $\mathbf{P}_{\mathbf{I}}$ & $\mathbf{P}_{\mathbf{2}}$ & $\mathbf{P}_{3}$ \\
\hline Age, years & $58.9 \pm 11.3$ & $58.0 \pm 11.1$ & $58.6 \pm 11.6$ & $59.7 \pm 11.2$ & $<0.001$ & $<0.001$ & 0.001 & $<0.001$ \\
\hline Sex, male & $13,156(54.2)$ & $2390(53.2)$ & $5579(53.4)$ & $5187(55.7)$ & $<0.001$ & 0.841 & 0.007 & 0.001 \\
\hline Diabetes duration, years & $4.2(2.0-8.4)$ & $3.9(1.8-7.4)$ & $4.2(1.6-9.0)$ & $4.4(2.3-8.4)$ & $<0.001$ & $<0.001$ & $<0.001$ & $<0.001$ \\
\hline \multicolumn{9}{|l|}{ Median (IQR) } \\
\hline $\mathrm{BMI}, \mathrm{Kg} / \mathrm{m}^{2}$ & $24.2 \pm 3.0$ & $23.9 \pm 3.0$ & $24.1 \pm 3.0$ & $24.5 \pm 3.0$ & $<0.001$ & 0.010 & $<0.0 .01$ & $<0.01$ \\
\hline FPG at diagnosis, mmol/L & $8.2 \pm 2.6$ & $8.5 \pm 1.4$ & $6.3 \pm 0.8$ & $10.3 \pm 2.6$ & $<0.001$ & $<0.001$ & $<0.001$ & $<0.001$ \\
\hline 2h-PPG at diagnosis, $\mathrm{mmol} / \mathrm{L}$ & $13.8 \pm 3.5$ & $9.7 \pm 1.3$ & $14.0 \pm 2.5$ & $15.5 \pm 3.6$ & $<0.001$ & $<0.001$ & $<0.001$ & $<0.001$ \\
\hline $\mathrm{HbAIc}, \%$ & $7.4 \pm 1.5$ & $7.3 \pm 1.3$ & $7.4 \pm 1.5$ & $7.8 \pm 1.6$ & $<0.001$ & $<0.001$ & $<0.001$ & $<0.001$ \\
\hline $\mathrm{HbAlc}$ control target & $6715(27.7)$ & $1474(32.8)$ & $3042(29.1)$ & $2199(23.6)$ & $<0.001$ & $<0.001$ & $<0.001$ & $<0.001$ \\
\hline SBP, mmHg & $132.2 \pm 15.0$ & $|3| .| \pm| 4.6$ & $132.4 \pm 15.0$ & $132.5 \pm 15.2$ & $<0.001$ & $<0.001$ & $<0.001$ & 0.593 \\
\hline $\mathrm{DBP}, \mathrm{mmHg}$ & $81.9 \pm 10.9$ & $81.7 \pm 10.6$ & $81.8 \pm 11.0$ & $82.1 \pm 10.9$ & 0.129 & 0.484 & 0.063 & 0.135 \\
\hline Blood pressure control target & $|4,12|(58.9)$ & $2755(61.3)$ & $5848(55.9)$ & $5518(59.2)$ & $<0.001$ & $<0.001$ & 0.018 & $<0.001$ \\
\hline Self-monitoring of blood glucose & $9972(41.1)$ & $1631(36.3)$ & $4344(41.6)$ & $3997(42.9)$ & $<0.001$ & $<0.001$ & $<0.001$ & 0.059 \\
\hline Triglycerides, $\mathrm{mmol} / \mathrm{L}, \mathrm{mmol} / \mathrm{L}$ & $2.0 \pm 1.1$ & $2.1 \pm 1.4$ & $1.9 \pm 1.0$ & $2.5 \pm 1.1$ & $<0.001$ & $<0.001$ & $<0.001$ & $<0.001$ \\
\hline Low density lipoprotein cholesterol, $\mathrm{mmol} / \mathrm{L}$ & $2.8 \pm 1.0$ & $2.7 \pm 1.0$ & $2.8 \pm 0.9$ & $2.8 \pm 1.0$ & $<0.001$ & $<0.001$ & $<0.001$ & 0.918 \\
\hline Total cholesterol, mmol/L & $4.8 \pm 1.4$ & $4.9 \pm 1.7$ & $4.7 \pm 1.4$ & $4.9 \pm 1.3$ & $<0.001$ & $<0.001$ & 0.620 & $<0.001$ \\
\hline Lipid target & $5942(24.5)$ & $1177(26.2)$ & $2498(23.9)$ & $2267(24.3)$ & $<0.001$ & 0.003 & 0.017 & 0.484 \\
\hline \multicolumn{9}{|l|}{ Diabetes treatment } \\
\hline OAD only & $13,580(56.0)$ & $2674(59.5)$ & $6462(61.8)$ & 4444(47.7) & $<0.001$ & $<0.001$ & $<0.001$ & $<0.001$ \\
\hline OAD plus insulin & $5185(21.4)$ & $780(17.4)$ & 1976(18.9) & $2429(26.1)$ & $<0.001$ & $<0.001$ & $<0.001$ & $<0.001$ \\
\hline OAD plus GLP-I & $35(0.1)$ & $8(0.2)$ & $9(0.1)$ & $18(0.2)$ & 0.113 & 0.126 & 0.848 & 0.042 \\
\hline Insulin only & $5054(20.8)$ & $954(21.2)$ & $1772(17.0)$ & $2328(25.0)$ & $<0.001$ & $<0.001$ & $<0.001$ & $<0.001$ \\
\hline GLP-I only & $31(0.1)$ & $9(0.2)$ & $10(0.1)$ & $12(0.1)$ & 0.259 & 0.100 & 0.312 & 0.486 \\
\hline Lifestyle only & $381(1.6)$ & $68(1.5)$ & $224(2.1)$ & $89(0.9)$ & $<0.001$ & $<0.001$ & $<0.001$ & $<0.001$ \\
\hline \multicolumn{9}{|l|}{ Economic development level } \\
\hline Underdeveloped & $3516(14.5)$ & $482(10.7)$ & |444(|3.8) & $1590(17.1)$ & $<0.001$ & $<0.001$ & $<0.001$ & $<0.001$ \\
\hline Intermediately developed & $1997(16.5)$ & $749(16.7)$ & $1566(15.0)$ & $1682(18.0)$ & $<0.001$ & 0.009 & 0.047 & $<0.001$ \\
\hline Developed & $16,753(69.0)$ & $3262(72.6)$ & $7443(71.2)$ & $6048(64.9)$ & $<0.001$ & 0.082 & $<0.001$ & $<0.001$ \\
\hline \multicolumn{9}{|l|}{ Macrovascular complications } \\
\hline Isolated coronary heart disease & $2226(9.2)$ & $332(7.4)$ & $1073(10.3)$ & $821(8.8)$ & $<0.001$ & $<0.001$ & 0.005 & 0.001 \\
\hline Isolated cerebrovascular disease & $758(3.1)$ & $100(2.2)$ & $322(3.1)$ & $336(3.6)$ & $<0.001$ & 0.004 & $<0.001$ & 0.040 \\
\hline $\begin{array}{l}\text { Combined cardiovascular and cerebrovascular } \\
\text { disease }\end{array}$ & $478(2.0)$ & $58(1.3)$ & $231(2.2)$ & $189(2.0)$ & 0.001 & $<0.001$ & 0.002 & 0.376 \\
\hline \multicolumn{9}{|l|}{ Microvascular complications } \\
\hline IDR & $1435(5.9)$ & $230(5.1)$ & $580(5.6)$ & $625(6.7)$ & $<0.001$ & 0.288 & $<0.001$ & 0.001 \\
\hline IDN & $781(3.2)$ & $99(2.2)$ & $322(3.1)$ & $360(3.9)$ & $<0.001$ & 0.003 & $<0.001$ & 0.003 \\
\hline CRN & $533(2.2)$ & $58(1.3)$ & $207(2.0)$ & $268(2.9)$ & $<0.001$ & 0.003 & $<0.001$ & $<0.001$ \\
\hline
\end{tabular}

Notes: Data were expressed as mean \pm standard deviation or $\mathrm{n}(\%)$, unless otherwise indicated. Blood pressure target was defined as < $40 / 90 \mathrm{mmHg}$. Lipid target was defined as TG $<1.7 \mathrm{mmol} / \mathrm{L}$ and LDL-C $<2.6 \mathrm{mmo} / \mathrm{L}$. $\mathrm{P}$ value was derived from ANOVA, $\chi^{2}$ test, Fisher's exact test or Kruskal-Wallis rank test among three groups. $\mathrm{P}_{1}$ value was for pairwise comparison between IFH and IPH. $\mathrm{P}_{2}$ value was for pairwise comparison between IFH and $\mathrm{CH}$. $\mathrm{P}_{3}$ value was for pairwise comparison between IPH and $\mathrm{CH}$.

Abbreviations: IQR, interquartile range; IFH, isolated fasting hyperglycemia; IPH, isolated postprandial hyperglycemia; $\mathrm{CH}$, combined hyperglycemia; OAD, oral antidiabetic drugs. GLP-I, glucagon-like peptide-I; IDR, isolated diabetic retinopathy; IDN, isolated diabetic nephropathy; CRN, combined diabetic retinopathy and nephropathy. 
were drawn by Graphpad Prism 7 (Graphpad Software Company, CA, USA).

\section{Results}

\section{Clinical Characteristics of T2DM}

\section{Participants}

Among the 24,266 participants included in this study, the mean age was 58.9 years (SD 11.3 years). About $54 \%$ of participants were male. The number of IFH participants, IPH participants, CH participants was 4493 (18.5\%), 10,453 (43.1\%) and 9320 (38.4\%), respectively. The participants in $\mathrm{CH}$ group were more likely to be older; had slightly higher BMI, triglyceride, glucose indices and longer duration of diabetes; had higher proportion of treatment with OADs plus insulin and treatment with insulin only; and had a lower proportion of participants in economically developed region, blood pressure control target and HbAlc control target (Table 1).

\section{Cumulative Incidence of Microvascular Diseases Among T2DM Participants with Different Abnormal Glycemic Phenotypes} In different phenotype groups of IFH, IPH and $\mathrm{CH}$, the cumulative incidences of IDR were $5.1 \%, 5.5 \%$ and $6.7 \%$, the cumulative incidences of IDN were $2.2 \%, 3.1 \%$ and $3.9 \%$, and the cumulative incidences of CRN were $1.3 \%$, $2.0 \%$ and $2.9 \%$ (Table 1). Kaplan-Meier curves in three phenotype groups appeared slightly different within diabetes duration of 5 years and started to separate and continued to diverge with increasing diabetes duration (Figure 1). Participants with $\mathrm{CH}$ phenotype showed the highest cumulative incidences of IDR, IDN and CRN, followed by IPH phenotype ( $\log$ rank test $\mathrm{P}<0.001$ ). Through pairwise comparison, the phenotype of IPH showed a significantly higher cumulative incidence of IDR, IDN and CRN than IFH phenotype group, but lower cumulative incidence compared to those in the $\mathrm{CH}$ phenotype group (All P for pairwise comparison $<0.001$ ).

\section{Association Between Abnormal Glycemic Phenotypes and Microvascular Diseases in T2DM Participants}

In the multivariable-adjusted model, after step forward adjustment for age, sex, diabetes duration, BMI, selfmonitoring of blood glucose, diabetes treatment, the target of blood pressure control, the target of lipid control, $\mathrm{HbA1c}$ and economic development level, the results were similar across different models. The full adjusted covariate model, using the phenotype of IFH as reference, showed
A

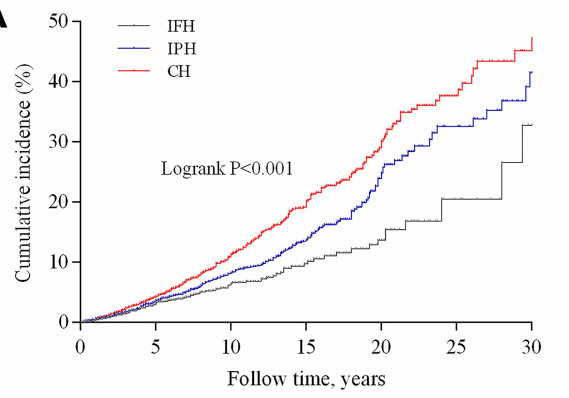

B

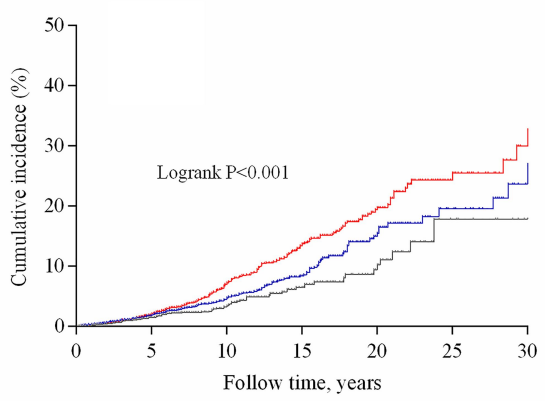

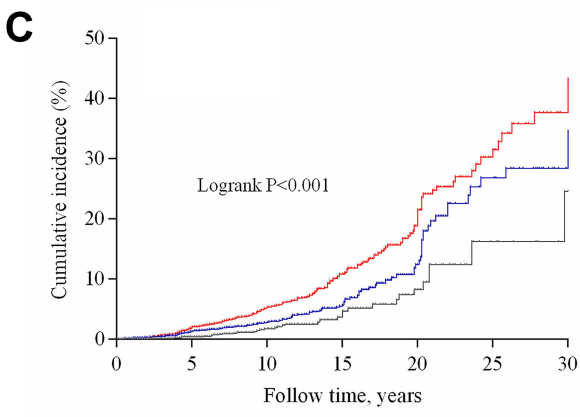

Figure I Cumulative incidence of microvascular diseases stratified by abnormal glycemic phenotype for T2DM participants: (A) isolated diabetic retinopathy (IDR), (B) isolated diabetic nephropathy (IDN), (C) combined diabetic retinopathy and nephropathy (CRN). Log rank test for microvascular diseases of T2DM participants with different abnormal glycemic phenotype (all P<0.05 in pairwise comparison among three phenotypes: IDR, IDN and CRN). 
that participants with IPH phenotype presented a significantly higher risk of IDR (OR: 1.16, 95\% CI: 1.05-1.28), IDN (OR: 1.37, 95\% CI: 1.09-1.37) and CRN (OR:1.64, 95\% CI: 1.21-2.21). Understandably, the phenotype of $\mathrm{CH}$ was associated with the highest risk of IDR (OR: 1.20; 95\% CI: 1.02-1.41), IDN (OR: 1.59; 95\% CI: 1.27-2.01) and CRN (OR: 1.93, 95\% CI: 1.44-2.59) (Table 2). After stratifying the participants by age, sex, duration of diabetes, and BMI, the subgroup analysis still indicated similar results of the higher risks of IDR, IDN and CRN in IPH compared with the phenotype of IFH (Figure 2).

\section{Discussion}

In the present study, we demonstrated that participants with IPH phenotype had higher cumulative occurrence and onset risks of IDR, IDN and CRN compared with the participants with IFH phenotype, but lower cumulative onset risks and onset risks of IDR, IDN and CRN compared with the $\mathrm{CH}$ phenotype group. These results further occurred in the subgroup analysis stratified by age, sex, duration of diabetes and BMI. To the best of our knowledge, this is the first population-based study to demonstrate the association between abnormal glycemic phenotypes and the onset risk of diabetic microvascular complications in Chinese T2DM population.

Several reasons might account for the higher risk of microvascular complications in IPH phenotype group in our study. Firstly, HbA1c elevation has been accepted as an independent risk factor for diabetic microvascular disease. Several clinical trials previously demonstrated that intensive glucose control (mostly HbA1c target $<7 \%$ ) can prevent the development or

Table 2 Association Between Abnormal Glycemic Phenotypes and Microvascular Disease in Type 2 Diabetes Mellitus Participants

\begin{tabular}{|c|c|c|c|}
\hline & IDR & IDN & CRN \\
\hline & Odds Ratio (95\% Cl) & Odds Ratio $(95 \% \mathrm{CI})$ & Odds Ratio (95\% Cl) \\
\hline \multicolumn{4}{|l|}{ Model I } \\
\hline IFH & I.00 (Reference) & I.00 (Reference) & I.00 (Reference) \\
\hline $\mathrm{IPH}$ & $1.23(1.07-1.40)$ & $1.43(1.14-1.80)$ & $1.57(1.17-2.10)$ \\
\hline $\mathrm{CH}$ & $1.38(1.18-2.62)$ & I.85 (I.48-2.32) & $2.35(1.77-2.13)$ \\
\hline Model fit (C) & $0.54 I$ & 0.555 & $0.577 z$ \\
\hline \multicolumn{4}{|l|}{ Model 2} \\
\hline IFH & I.00 (Reference) & I.00 (Reference) & I.00 (Reference) \\
\hline IPH & $1.14(1.05-1.25)$ & $1.35(1.08-1.70)$ & $1.46(1.08-1.95)$ \\
\hline $\mathrm{CH}$ & $1.37(1.17-1.60)$ & I.82 (I.45-2.28) & $2.30(1.73-3.06)$ \\
\hline Age, years & $1.03(1.02-1.03)$ & $1.04(1.03-1.04)$ & $1.04(1.04-1.04)$ \\
\hline Model fit (C) & 0.596 & 0.620 & 0.640 \\
\hline \multicolumn{4}{|l|}{ Model 3} \\
\hline IFH & 1.00 (Reference) & I.00 (Reference) & I.00 (Reference) \\
\hline $\mathrm{IPH}$ & I.II (I.03-I.2I) & I.31 (I.04-1.65) & $1.41(1.05-1.90)$ \\
\hline $\mathrm{CH}$ & $1.33(1.14-1.56)$ & I.77 (I.4I-2.22) & $2.24(1.67-2.30)$ \\
\hline Age, years & I.0I (I.00-1.02) & $1.02(1.01-1.03)$ & $1.02(1.01-1.03)$ \\
\hline Duration of diabetes, years & $1.09(1.08-1.10)$ & $1.09(1.07-1.10)$ & $1.12(1.11-1.14)$ \\
\hline Model fit (C) & 0.663 & 0.682 & 0.744 \\
\hline \multicolumn{4}{|l|}{ Model 4} \\
\hline IFH & I.00 (Reference) & I.00 (Reference) & I.00 (Reference) \\
\hline $\mathrm{IPH}$ & $1.16(1.05-1.28)$ & $1.37(1.09-1.73)$ & $\mathrm{I} .64(|.2|-2.2 \mid)$ \\
\hline $\mathrm{CH}$ & $\mathrm{I} .20(\mathrm{I} .02-1.4 \mathrm{I})$ & $1.59(1.27-2.01)$ & $1.93(1.44-2.59)$ \\
\hline Age, years & $1.01(1.01-1.02)$ & $1.02(1.01-1.03)$ & $1.02(1.01-1.03)$ \\
\hline Duration of diabetes, years & $1.08(1.07-1.09)$ & $1.08(1.07-1.09)$ & $1.11(1.10-1.13)$ \\
\hline Model fit (C) & 0.711 & 0.717 & 0.792 \\
\hline
\end{tabular}

Notes: Data are odds ratio $(95 \% \mathrm{Cl})$ from logistic regression model. Model I has no adjustment of covariable. Model 2 was adjusted for age, sex. Model 3 was adjusted for age, sex. Model 4 was adjusted for age, sex, BMI, self-monitoring of blood glucose, diabetes treatment, the target of blood pressure control, the target of lipid control, $\mathrm{HbAlc}$ and economic development level.

Abbreviations: IFH, isolated fasting hyperglycemia; IPH, isolated postprandial hyperglycemia; $\mathrm{CH}$, combined hyperglycemia; IDR, isolated diabetic retinopathy; IDN, isolated diabetic nephropathy; CRN, combined diabetic retinopathy and nephropathy; $\mathrm{Cl}$, confidence interval. 


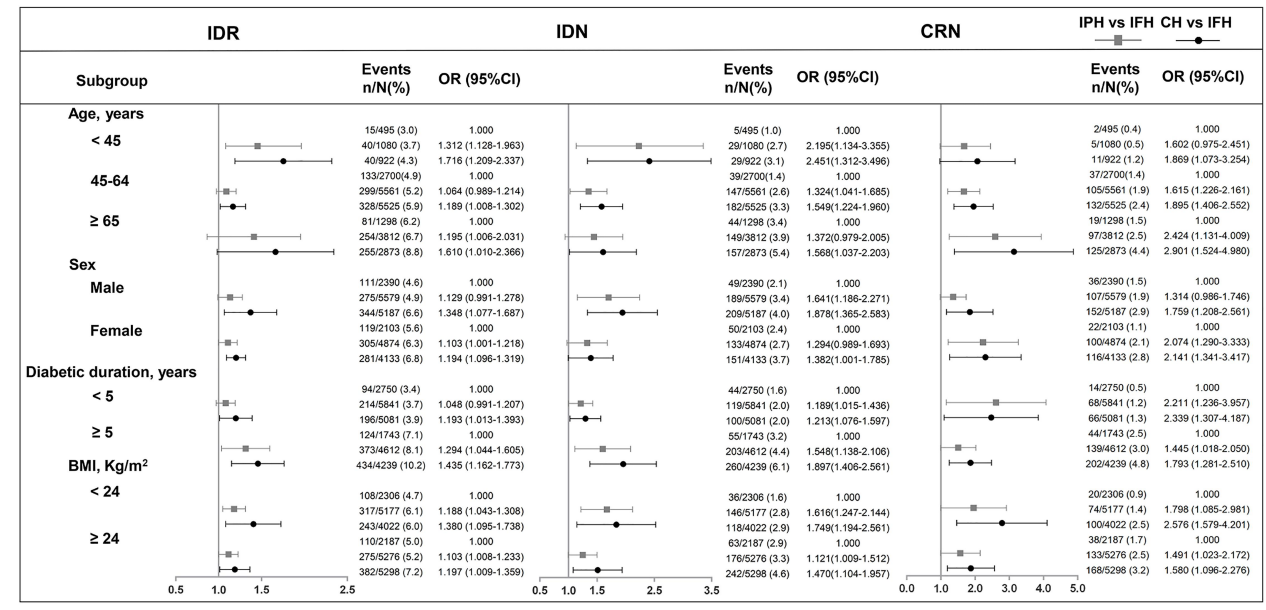

Figure 2 Subgroup analysis of association between abnormal glycemic phenotypes and microvascular complication for type 2 diabetes mellitus participants. Abbreviations: IDR, isolated diabetic retinopathy; IDN, isolated diabetic nephropathy; CRN, combined diabetic retinopathy and nephropathy; IFH, isolated fasting hyperglycemia; IPH, isolated postprandial hyperglycemia; $\mathrm{CH}$, combined hyperglycemia; $\mathrm{BMI}$, body mass index; OR, odds ratio; $\mathrm{Cl}$, confidence interval.

slow down the progress of microvascular complications. ${ }^{17-19}$ It was reported that postprandial glucose exceeded $50 \%$ or fasting glucose for the contribution of $\mathrm{HbAl} \mathrm{c}$ when $\mathrm{HbAlc}<$ $8.4 \%$ or $5.1 \%-7.4 \%$, respectively. ${ }^{20}$ Furthermore, compared with Caucasians, postprandial blood glucose has a more prominent contribution to $\mathrm{HbA} 1 \mathrm{c}$ for Chinese or Asian people. ${ }^{21}$ A national diabetes epidemiology survey showed nearly half of newly diagnosed diabetes patients had IPH phenotype in China. ${ }^{22}$ A meta-analysis also reported that PPG had a closer association with $\mathrm{HbAlc}$ than FPG, and had a better predictive performance in predicting overall glycemic control. ${ }^{23}$ As a result, we speculated that intensive HbA1c control caused by decreasing postprandial glucose would obtain more benefit in preventing microvascular disease. Additionally, the mean level of HbA1c was $7.4 \%$ in our study, and the participants with IPH phenotype had a worse HbA1c, so the IPH phenotype group presented a greater risk of microvascular complications in this study. In addition, postprandial hyperglycemia is characterized by a rapid and large increase in blood glucose concentrations, which produce an increase in glomerular filtration rate in diabetic patients. ${ }^{24}$ The hyperperfusion results in a greater stimulus for hyperproduction of collagen which was considered as an important event for pathogenesis of diabetic nephropathy. ${ }^{25}$ Simultaneously, blood flow was also closely parallel with plasma glucose concentrations, ${ }^{26}$ which are both pathogenetic factors that are important in the occurrence and progression of retinopathy in diabetic patients. ${ }^{27}$ For $\mathrm{CH}$ phenotype, T2DM patients presented the characteristic of combined fasting and postprandial hyperglycemia, so it had the worst HbAlc control and the highest risks of IDR, IDN or CRN.
The strength of our study is the relatively large sample size, and the participants were recruited from almost all the provinces of China, representing people with T2DM throughout China to some extent. Moreover, the targeted study population was proportionally randomly selected using a three-stage stratification-random clustering sampling method, resulting in the utmost reduction of selection bias. However, there were several limitations in this study. Firstly, diabetes complication cases were ascertained by reviewing medical records, not by systematical screening. Some medical records were not exactly available and therefore some microvascular disease cases might have been missed, especially for T2DM participants in economically disadvantaged regions. As a result, the true prevalence of diabetes complication might have been underestimated. Secondly, lifestyle information such as smoking, drinking, physical exercise and dietary intake, which are associated with vascular disease, was not collected. Thirdly, the study design was cross-sectional, so competing risks could not be considered, and the causal association between phenotypes and complication was not investigated. Fourth, considering the feasibility of the study, the sampling approach of including the first 7 consecutive patients was not completely random, which may have led to bias.

\section{Conclusion}

In summary, our data suggest that T2DM participants with IPH phenotype have higher risk of microvascular disease than T2DM patients with IFH phenotype. Through analyzing the differences in the risks of microvascular diseases including IDR, IDN, and CRN, in participants with abnormal glycemic 
phenotypes, this study could help endocrinologists better identify the high-risk population. Special focus should be directed on T2DM patients with IPH phenotype for the prevention of microvascular diseases in the early stage of diabetes.

\section{Acknowledgments}

We appreciate the support of Novo Nordisk Company (Beijing, China) and the efforts of all participants who contributed to the study design and data collection. This study was supported by grant of National Natural Science Foundation of China (31672375) to Yan He. All authors agreed to the content of the manuscript and its submission for publication.

\section{Disclosure}

The authors report no conflicts of interest in this work.

\section{References}

1. Rathmann W, Giani G. Global Prevalence of Diabetes: estimates for the Year 2000 and Projections for 2030: response to Wild et al.. Diabetes Care. 2004;27(10):2568-2569. doi:10.2337/diacare.27.10.2568

2. Wang L, Gao P, Zhang M, et al. Prevalence and Ethnic Pattern of Diabetes and Prediabetes in China in 2013. JAMA. 2017;317 (24):2515-2523. doi:10.1001/jama.2017.7596

3. Whiting DR, Guariguata L, Weil C, et al. IDF diabetes atlas: global estimates of the prevalence of diabetes for 2011 and 2030. Diabetes Res Clin Pract. 2011;94(3):311-321. doi:10.1016/j.diabres.2011.10.029

4. Go AS, Chertow GM, Fan D, et al. Chronic kidney disease and the risks of death, cardiovascular events, and hospitalization. $N$ Engl J Med. 2004;351(13):1296-1305. doi:10.1056/NEJMoa041031

5. Cheung N, Mitchell P, Wong TY. Diabetic retinopathy. Lancet. 2010;376(9735):124-136. doi:10.1016/S0140-6736(09)62124-3

6. Yau JW, Rogers SL, Kawasaki R, et al. Global prevalence and major risk factors of diabetic retinopathy. Diabetes Care. 2012;35 (3):556-564. doi:10.2337/dc11-1909

7. Gabir MM, Hanson RL, Dabelea D, et al. Plasma glucose and prediction of microvascular disease and mortality: evaluation of 1997 American Diabetes Association and 1999 World Health Organization criteria for diagnosis of diabetes. Diabetes Care. 2000;23 (8):1113-1118. doi:10.2337/diacare.23.8.1113

8. Liu Y, Yang J, Tao L, et al. Risk factors of diabetic retinopathy and sight-threatening diabetic retinopathy: a cross-sectional study of 13 473 patients with type 2 diabetes mellitus in mainland China. BMJ Open. 2017;7(9):e016280. doi:10.1136/bmjopen-2017-016280

9. Patel YR, Kirkman MS, Considine RV, et al. Retinopathy predicts progression of fasting plasma glucose: an Early Diabetes Intervention Program (EDIP) analysis. J Diabetes Complications. 2017;31 (3):605-610. doi:10.1016/j.jdiacomp.2016.11.005

10. Shiraiwa T, Kaneto H, Miyatsuka T, et al. Postprandial hyperglycemia is a better predictor of the progression of diabetic retinopathy than $\mathrm{HbAlc}$ in Japanese type 2 diabetic patients. Diabetes Care. 2005;28(11):2806-2807. doi:10.2337/diacare.28.11.2806

11. Hsieh M-H, Yang J-F, Lin W-Y, et al. Fasting sugar, blood pressure, and uric acid are factors related to positive proteinuria and an impaired eGFR. $J$ Chin Med Assoc. 2017;80(12):782-789. doi:10.1016/j.jcma.2016.11.011
12. Shichiri M, Kishikawa H, Ohkubo Y, et al. Long-term results of the Kumamoto Study on optimal diabetes control in type 2 diabetic patients. Diabetes Care. 2000;23(Suppl 2):B21-29.

13. Vieira MB, Neves JS, Leitão L, et al. Impaired Fasting Glucose and Chronic Kidney Disease, Albuminuria, or Worsening Kidney Function: A Secondary Analysis of SPRINT. J Clin Endocrinol Metab. 2019;104(9):4024-4032. doi:10.1210/jc.2019-00073

14. McCane DR, Hanson RL, Charles MA, et al. Comparison of tests for glycated haemoglobin and fasting and two hour plasma glucose concentrations as diagnostic methods for diabetes. BMJ. 1994;308 (6940):1323-1328. doi:10.1136/bmj.308.6940.1323

15. Liu G, Dou J, Pan Y, et al. Comparison of the Effect of Glycemic Control in Type 2 Diabetes Outpatients Treated With Premixed and Basal Insulin Monotherapy in China. Front Endocrinol (Lausanne). 2018;9:639. doi:10.3389/fendo.2018.00639

16. Alberti KG, Zimmet PZ. Definition, diagnosis and classification of diabetes mellitus and its complications. Part 1: diagnosis and classification of diabetes mellitus. Provisional report of a WHO Consultation. Diabetic Med. 1998;15(7):539-553. doi:10.1002/ (SICI)1096-9136(199807)15:7<539::AID-DIA668>3.0.CO;2-S

17. Hayward RA, Reaven PD, Wiitala WL, et al. Follow-up of glycemic control and cardiovascular outcomes in type 2 diabetes. $N$ Engl J Med. 2015;372(23):2197-2206. doi:10.1056/NEJMoa1414266

18. Holman RR, Paul SK, Bethel MA, et al. 10-year follow-up of intensive glucose control in type 2 diabetes. $N$ Engl J Med. 2008;359 (15):1577-1589. doi:10.1056/NEJMoa0806470

19. Patel A, MacMahon S, Chalmers J, et al. Intensive blood glucose control and vascular outcomes in patients with type 2 diabetes. N Engl J Med. 2008;358(24):2560-2572.

20. Monnier L, Lapinski H, Colette C. Contributions of Fasting and Postprandial Plasma Glucose Increments to the Overall Diurnal Hyperglycemia of Type 2 Diabetic Patients: variations with increasing levels of HbAlc. Diabetes Care. 2003;26(3):881-885. doi: $10.2337 /$ diacare. 26.3 .881

21. UK Prospective Diabetes Study (UKPDS) Group. Intensive blood-glucose control with sulphonylureas or insulin compared with conventional treatment and risk of complications in patients with type 2 diabetes (UKPDS 33). Lancet. 1998;352(9131):837-853. doi:10.1016/S0140-6736(98)07019-6

22. Yang W, Lu J, Weng J, et al. Prevalence of diabetes among men and women in China. $N$ Engl J Med. 2010;362(12):1090-1101. doi:10.1056/NEJMoa0908292

23. Ketema EB, Kibret KT. Correlation of fasting and postprandial plasma glucose with HbA1c in assessing glycemic control; systematic review and meta-analysis. Arch Public Health. 2015;73(1):43. doi:10.1186/s13690-015-0088-6

24. Skott P, Vaag A, Hother-Nielsen O, et al. Effects of hyperglycaemia on kidney function, atrial natriuretic factor and plasma renin in patients with insulin-dependent diabetes mellitus. Scand J Clin Lab Invest. 1991;51(8):715-727.

25. Steffes MW, Bilous RW, Sutherland DE, et al. Cell and matrix components of the glomerular mesangium in type I diabetes. Diabetes. 1992;41(6):679-684. doi:10.2337/diab.41.6.679

26. Rassam SM, Patel V, Kohner EM. The effect of experimental hypertension on retinal vascular autoregulation in humans: a mechanism for the progression of diabetic retinopathy. Exp Physiol. 1995;80 (1):53-68. doi:10.1113/expphysiol.1995.sp003834

27. Kohner EM, Patel V, Rassam SM. Role of blood flow and impaired autoregulation in the pathogenesis of diabetic retinopathy. Diabetes. 1995;44(6):603-607. doi:10.2337/diab.44.6.603 
Diabetes, Metabolic Syndrome and Obesity: Targets and Therapy is an international, peer-reviewed open-access journal committed to the rapid publication of the latest laboratory and clinical findings in the fields of diabetes, metabolic syndrome and obesity research. Origina research, review, case reports, hypothesis formation, expert opinion and commentaries are all considered for publication. The manuscript management system is completely online and includes a very quick and fair peer-review system, which is all easy to use. Visit http://www.dovepress.com/testimonials.php to read real quotes from published authors.

Submit your manuscript here: https://www.dovepress.com/diabetes-metabolic-syndrome-and-obesity-targets-and-therapy-journal 